\title{
Eating for two? Maternal weight gain and offspring cardiovascular risk
}

Gaining an excessive amount of weight before and during pregnancy could detrimentally affect the cardiovascular health of the child. This finding, from the Avon Longitudinal Study of Parents and Children (ALSPAC) is reported in Circulation by Abigail Fraser and colleagues.

Recruitment of pregnant women for this population-based birth cohort was conducted in 1991-1992, and the health of these mothers and their offspring continues to be assessed. "ALSPAC is unique in that mothers had weight repeatedly measured during pregnancy," says Dr Fraser; "nowadays this is not the practice in the UK and many other European countries."

The investigators found that women who gained more weight than is recommended in the 2009 guidelines from the US Institute of Medicine tended to have offspring with increased BMI, systolic blood pressure, fat mass, and waist circumference. These children also had higher levels of C-reactive protein, interleukin 6, and leptin, as well as lower levels of HDL cholesterol and apolipoprotein A-I than children born to mothers who did not gain more than the recommended amount of weight. A continuous association existed between maternal weight gain during the first 14 weeks of gestation and offspring adiposity. The researchers also found that the children of women who are overweight before they conceive are more likely to develop cardiovascular risk factors than the children of women who are not overweight. According to Dr Fraser, this finding emphasizes "the importance of initiatives aimed at encouraging women in their reproductive years to maintain a healthy weight".

Alexandra King

Original article Fraser, A. et al. Association of maternal weight gain in pregnancy with offspring obesity and metabolic and vascular traits in childhood. Circulation 121, 2557-2564 (2010) 